\title{
In-trap decay spectroscopy with the TITAN facility at TRIUMF
}

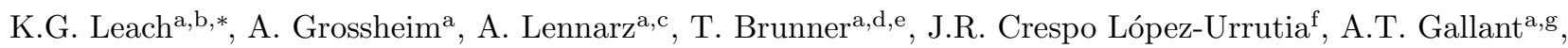
M. Good ${ }^{\mathrm{a}}$, R. Klawitter ${ }^{\mathrm{a}, \mathrm{f}}$, A.A. Kwiatkowski ${ }^{\mathrm{a}}$, T. Ma ${ }^{\mathrm{b}}$, T.D. Macdonald ${ }^{\mathrm{a}, \mathrm{g}}$, S. Seeraji ${ }^{\mathrm{b}}$, M.C. Simon ${ }^{\mathrm{a}, 1}$, C. Andreoiu ${ }^{\mathrm{b}}$, J. Dilling ${ }^{\mathrm{a}, \mathrm{g}}$, D. Frekers ${ }^{\mathrm{c}}$

\author{
${ }^{a}$ TRIUMF, 4004 Wesbrook Mall, Vancouver, British Columbia V6T 2A3, Canada \\ ${ }^{b}$ Department of Chemistry, Simon Fraser University, Burnaby, British Columbia V5A 1S6, Canada \\ ${ }^{c}$ Institut für Kernphysik, Westfälische-Wilhelms-Universität Münster, D-48149 Münster, Germany \\ ${ }^{d}$ Physik Department E12, Technische Universität München, D-85748 Garching, Germany \\ ${ }^{e}$ Department of Physics, Stanford University, Stanford, CA 94305, USA

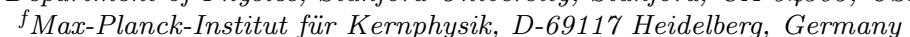 \\ ${ }^{g}$ Department of Physics and Astronomy, University of British Columbia, Vancouver, BC, V6T 1Z1, Canada
}

\section{Abstract}

A novel in-trap decay spectroscopy facility has been developed and constructed for use with TRIUMF's Ion Trap for Atomic and Nuclear science (TITAN). This apparatus consists of an open-access spectroscopy ion-trap, which is surrounded radially with up to seven low-energy planar $\mathrm{Si}(\mathrm{Li})$ detectors. The ion-trap environment allows for the detection of low-energy photons by providing backing-free storage of the radioactive ions, while guiding decay particles from the trap center via the strong (up to $6 \mathrm{~T}$ ) magnetic field. Excellent ion confinement is also facilitated by the use of an intense electron beam which provides storage times of minutes or more without ion loss. These advantages, along with careful monitoring and control, provide a significant increase in sensitivity for the detection of X-rays from the electron-capture process. The design, development, and commissioning of this apparatus are presented and the future of the device and experimental technique are discussed.

Keywords: in-trap decay spectroscopy, electron capture, beta-decay of highly-charged ions, X-ray detection, electron-beam ion trap, nuclear matrix elements, double beta decay

\section{Introduction}

\subsection{Nuclear matrix elements for $\beta \beta$ decay}

The relatively recent evidence that neutrinos have mass has generated a great deal of interest in exotic nuclear decay modes [1, 2]. As a part of these studies, searches for the $0 \nu$ mode of $\beta \beta$ decay is among the most relevant [2], since it violates lepton-number conservation and would establish the neutrino as a Majorana particle [3, 4]. If this decay mode is observed, the effective Majorana mass of

\footnotetext{
* Corresponding author

Email address: kleach@triumf.ca (K.G. Leach)

${ }^{1}$ Present address: Stefan Meyer Institute for Subatomic Physics, 1090 Vienna, Austria
}

the neutrino, $\left\langle m_{\beta \beta}\right\rangle$, can be deduced from $0 \nu \beta \beta$ measurements,

$$
\left(T_{1 / 2}^{0 \nu}\right)^{-1}=G^{0 \nu}(Q, Z)\left|M^{0 \nu}\right|^{2}\left\langle m_{\beta \beta}\right\rangle^{2}
$$

where $T_{1 / 2}^{0 \nu}$ is the observed half-life of the $0 \nu \beta \beta$ decay and $G^{0 \nu}(Q, Z)$ is the phase-space factor. The term $M^{0 \nu}$ is the nuclear matrix element (NME) connecting the initial and final $0^{+}$states, which results entirely from theoretical calculations. The calculation of $\beta \beta$ decay NMEs is the source of current theoretical efforts and include several different model descriptions. The accuracy and precision of $\left\langle m_{\beta \beta}\right\rangle$ from Eqn. 1 is limited by the ability to calculate the NMEs. Therefore, constraints on these calculations are required from experimental data. 
Typically, the NME calculations are benchmarked to $2 \nu \beta \beta$ data [5] (a process allowed by the Standard Model) where the decay path proceeds through $1^{+}$states in the odd-odd intermediate nucleus [6]. Therefore, measurements of the $\beta^{-}$and electron-capture (EC) branching ratios of the intermediate nuclei in the $2 \nu \beta \beta$ process are directly relevant for capturing the nuclear-physics information required in the calculation of $M^{2 \nu}$. Typically the EC transitions are several orders of magnitude weaker than the dominant $\beta^{-}$decays from the same parent nucleus, making them difficult to detect. To circumvent this challenge, a low-background, high-sensitivity decay spectroscopy tool is required for measuring characteristic X-rays from weak EC decays [6, 7].

\subsection{High sensitivity decay spectroscopy}

The characterization of radioactive decay via photon detection is a key measurement method and is among the primary experimental techniques currently employed in nuclear physics. With the advancement of rare-isotope beam (RIB) facilities [8, access to increasingly exotic radioactive nuclei has become possible, allowing for a variety of decay experiments on short- and long-lived nuclei. Modern decay spectroscopy devices employ multiple detection systems for both charged particles and photons to further increase the sensitivity of the experiment, thus allowing for the observation of weak signals 9 .

In EC decay, the characteristic X-ray originates from the filling of the vacated atomic $K$-shell electron, and typically has an energy less than $100 \mathrm{keV}$. For the cases of interest to $\beta \beta$-decay studies specifically [6] the X-rays are much lower in energy, and are generally less than $40 \mathrm{keV}$. To observe weak EC branches at these energies, it is important that effects such as (i) implantation-material photonabsorption, (ii) positron-annihilation-induced background, (iii) charged-particle-generated bremstrahlung, and (iv) Compton backgrounds are minimized. The reduction of photon backgrounds at these energies requires a high level of con-

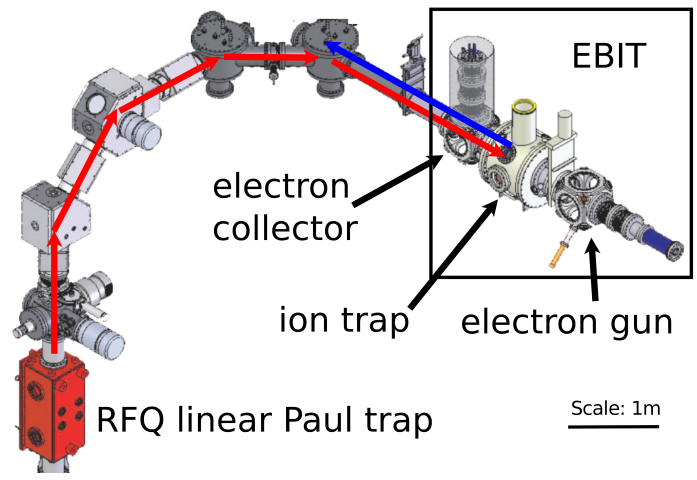

Figure 1: A schematic view of the TITAN facility at TRIUMF during decay-spectroscopy experiments. The ions are extracted in a bunch from the RFQ and injected as singly charged ions to the EBIT (path shown in red), where they are stored for charge breeding and decay spectroscopy. After the ions have been stored in the EBIT, they are extracted and dumped downstream away from the detectors (path shown in blue). The ion-bunch cycling is repeated continuously over the course of an experiment.

trol over the decay environment which can be provided using ion traps [10].

\subsection{In-trap decay studies}

The concept of observing decays from trapped radioactive species has been employed for years, most notably using magneto-optical traps (MOTs) and Paul traps, where charged particles and daughter recoils are detected to provide direct and indirect information about neutrinos [115], electrons [16, and neutrons [17. More recently, Penning traps have been considered to provide control over the decay environment [7, 18, 19], where charged particles can be directed along the magnetic field lines. Extending this concept, a high-sensitivity, low-background array of photon detectors was designed and constructed for use around an electron-beam ion trap (EBIT) at the TITAN facility at TRIUMF. The development, operational performance, commissioning, and future plans of this apparatus are described in the sections below. 


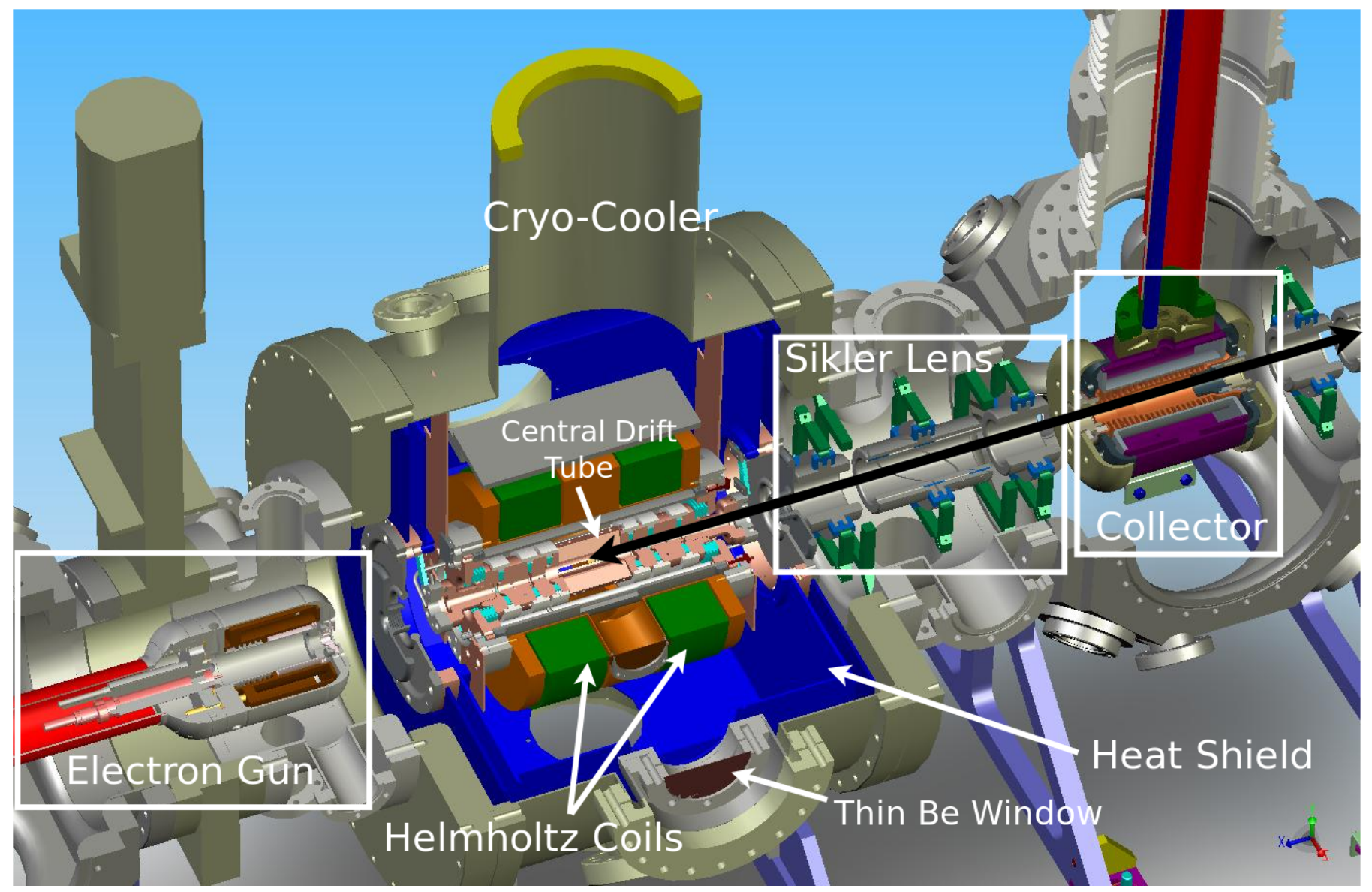

Figure 2: A technical depiction of the TITAN EBIT. The access ports on the outside of the trap house low-energy photon detectors and are separated from the trap volume by thin Be windows. The ion-bunch trajectories are schematically depicted here by the black double-arrow. The individual components are discussed further in the text.

\section{Experimental Facility}

\subsection{TRIUMF-ISAC}

The Isotope Separator and Accelerator (ISAC) facility [20, 21] at TRIUMF in Vancouver, Canada, employs a high-intensity (up to $100 \mu \mathrm{A}$ ) beam of $500 \mathrm{MeV}$ protons to produce RIBs using the isotope separation on-line (ISOL) technique [8, 22. ISAC is currently able to provide a wide variety of RIBs through the use of several different production target and ion-source combinations 23, including the recent use of uranium-carbide $\left(U C_{x}\right)$ targets [23]. This access to increasingly neutron-rich nuclei will be extended with the addition of the Advanced Rare-IsotopE Laboratory (ARIEL) 24].

Following the in-target production and ionization, the ions are mass separated before being delivered to the ex- perimental hall. The mass-selected, continuous beam of radioactive singly charged ions (SCIs) is delivered at low energies $(<60 \mathrm{keV})$ to a suite of experimental facilities for both cooled- and stopped-beam experiments 23 . The multiple-ion-trap system used for the novel decay-spectroscopy setup described in this article employs the use of a cooled and bunched beam, and is described in detail below.

\subsection{TITAN}

TRIUMF's Ion Trap for Atomic and Nuclear Science (TITAN) 25, 26] currently consists of three ion traps; (i) an RFQ linear Paul trap 27, 28 for buffer-gas cooling and bunching the low-energy ion beam, (ii) a $3.7 \mathrm{~T}$, highprecision mass-measurement Penning-trap (MPET) 29, and (iii) an electron-beam ion trap (EBIT) which provides 


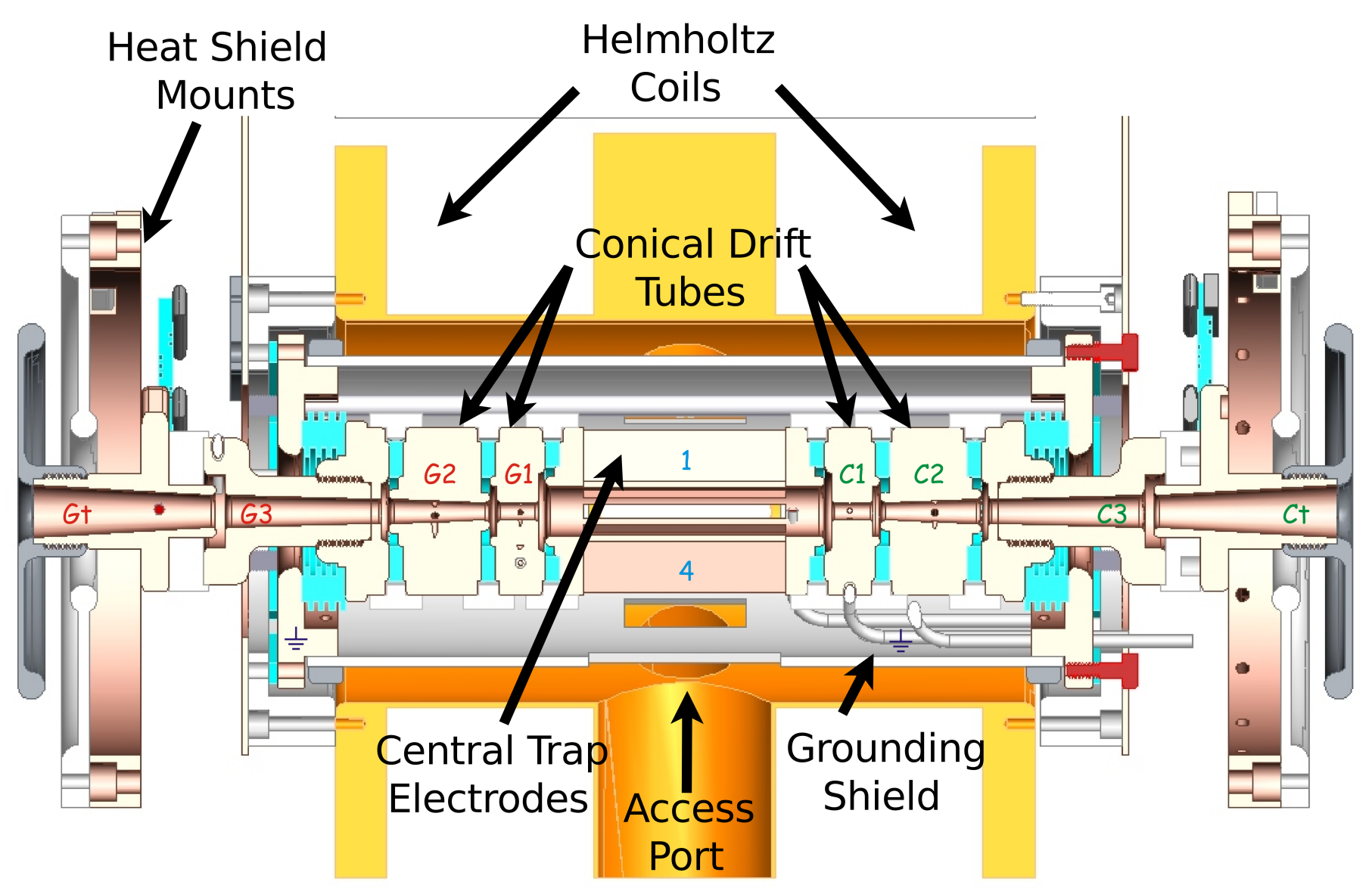

Figure 3: Cross-sectional view of the trap electrodes on both the gun $(G)$ and collector $(C)$ sides of the EBIT. The ion bunch is injected and extracted from the collector side of the trap by applying voltage to the four segments of the conical drift tubes (the trumpet $t$, and the three inner electrodes). When the ion bunch is trapped, it forms a prolate spheroidal shape and occupies the $7 \mathrm{~cm}$ axial volume of the eight-fold radially segmented central drift-tube. Segments 1 and 4 of the central trap electrodes are shown above.

highly charged ions (HCIs) 30.

In the near future, two components will be added to the TITAN system: (i) a cooler Penning-trap (CPET) to sympathetically cool HCIs with electrons or protons [31, and (ii) a multi-reflection time-of-flight (MR-ToF) device, for added beam purification [32. A schematic view of the TITAN facility at TRIUMF-ISAC is shown in Fig. 1 along with the ion path for typical decay-spectroscopy experiments.

\section{Decay Spectroscopy Ion Trap}

\subsection{Design specifications}

The TITAN EBIT (Fig. 2) is composed of a $500 \mathrm{~mA}$ electron gun2, a cold drift-tube assembly which is thermally coupled to a superconducting magnet, and an electron collector [30]. The drift-tube assembly of the trap (Fig. 3) is conically shaped, and the 8-fold radially segmented central electrode has an inner radius of $7.0 \mathrm{~mm}$ [30].

\subsection{Ion confinement}

The trapped ions are confined (i) axially by an electrostatic square-well potential, which is formed by apply-

\footnotetext{
${ }^{2}$ An upgrade for the electron gun is planned, which will allow for beam currents of up to $5 \mathrm{~A}$ in the near future.
} 


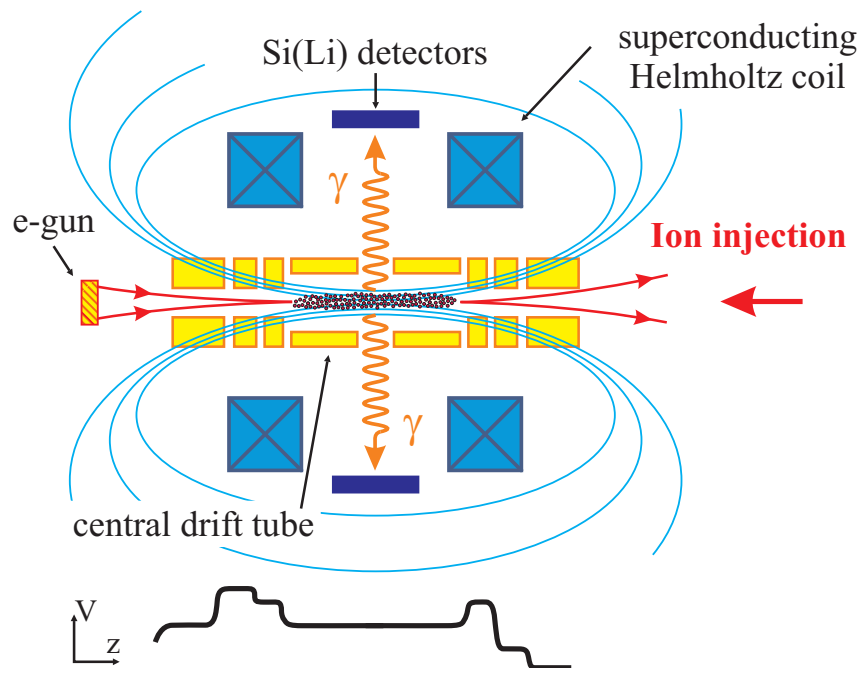

Figure 4: An illustration of the EBIT operation for decay spectroscopy and charge breeding (not to scale). Displayed in this diagram are: the trap electrodes, the magnetic coils and field lines, the ion injection/extraction site, the $e^{-}$beam, and the location of the photon detectors. Depicted below the trap components is a typical potential profile of the trap electrodes while the ion bunch is confined in the EBIT. The charged decay-particles are guided out of the trap by the magnetic field, while the photons emitted from the trapped ions are unimpeded.

ing voltage to the drift tubes that form the trap, and (ii) radially by the electron-beam space-charge potential and magnetic field [30]. Radial confinement of the ions in the central drift tube provides a spatial profile approximately equal to the radial extent of the electron beam. The axial confinement is defined by the length of the central drift tube, and forms a prolate spheroidal ion-cloud shape. A schematic diagram of the trapped ions in the EBIT is displayed in Fig. 4

Highly charged ions are generated in the EBIT by successive electron-impact ionization using a high-intensity electron beam compressed to a high charge density by a strong magnetic field. The up to 6 Tesla magnetic field is produced by two superconducting $\mathrm{Nb}_{3} \mathrm{Sn}$ coils in a Helmholtzlike configuration [30]. The simulated 33] and calculated 34] magnetic field strengths for this configuration are shown in Fig. 5. At the trap center, the field is reduced by $8 \%$ from this configuration, creating a magnetic bottle. $\beta$ particles that are emitted radially with an angle between the particle momentum and the axial magnetic field that is larger than the critical value stay trapped. The fraction of electrons emitted from the center of the trap that can escape via this process is calculated to be $77 \%$, which agrees with the results of the SIMION [35] simulations at $\vec{B}$-field strengths of 4,5 , and $6 \mathrm{~T}$. This mechanism, however, is not the sole contributing factor for charged decay particles to leave the trap. In fact, there are two secondary processes that enable the remaining particles to escape the trap volume:

1. The cyclotron frequency for $\beta$ particles in the EBIT is high ( $\approx 100 \mathrm{GHz}$ in a $4.5 \mathrm{~T}$ field $)$, and will therefore have many passes through the ion cloud, residual gas, and electron beam. As a result of this, the $\beta$-particles have a high probability for Coulomb scattering which causes them to drop below the critical angle and immediately escape the trap volume along the beam axis.

2. The transverse component of the $\beta$-particle velocity is cooled by synchrotron radiation, thereby decreasing the spiral radius to the point where it drops below the critical angle ${ }^{3}$

These processes are important for improving the photon detection sensitivity by removing charged-particle induced backgrounds, including guiding positrons from $\beta^{+}$ decay away from the trap center, thus providing $511 \mathrm{keV}$ suppression [36, 37].

\subsection{Trap access}

The EBIT features seven external ports, each with a $35.0 \mathrm{~mm}$ radius opening on the magnet housing, which allows for the mounting of photon detectors for performing spectroscopy on trapped ions. These ports are covered

\footnotetext{
${ }^{3}$ For example, a $5 \mathrm{MeV} \beta$ particle in a $4.5 \mathrm{~T}$ field experiences a typical cooling time of $\approx 20 \mathrm{~ms}$, which is, in general, much shorter than a typical trapping time for decay spectroscopy.
} 


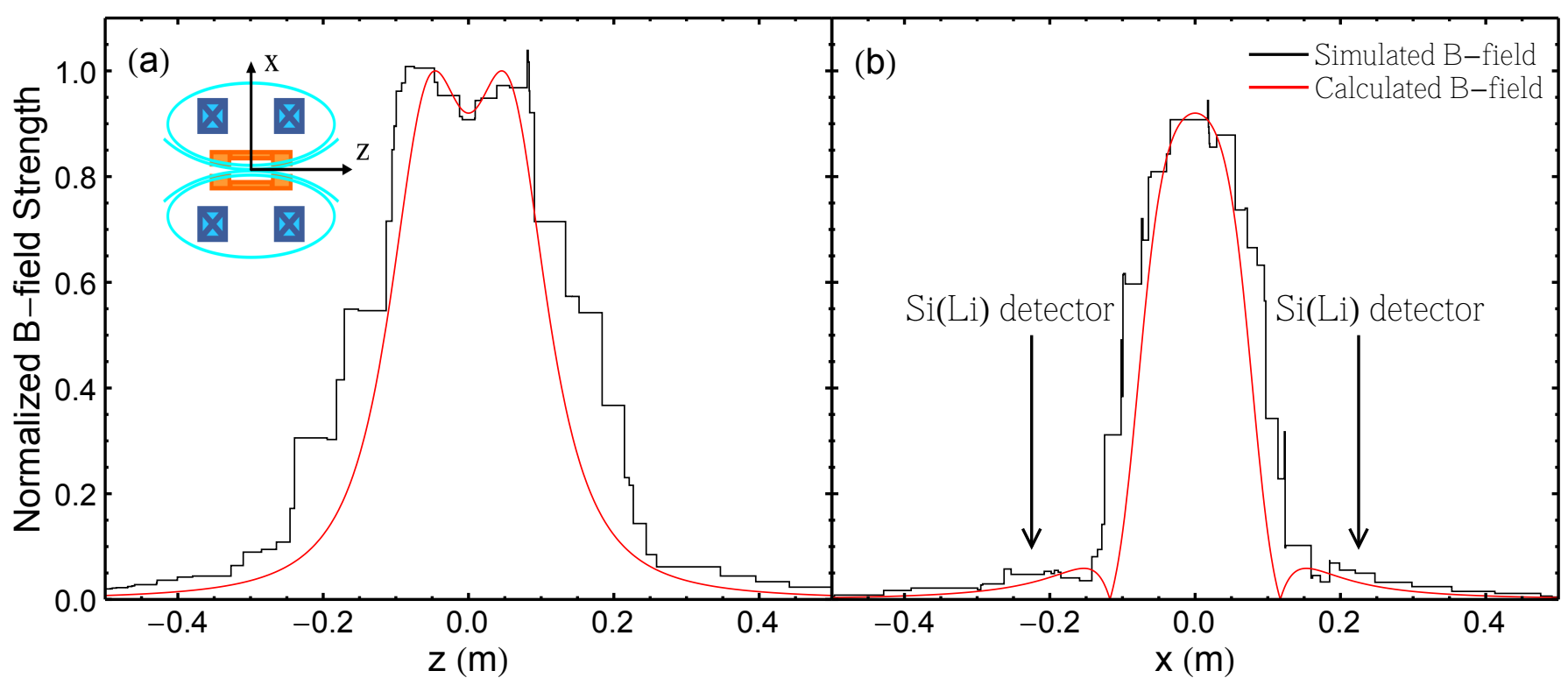

Figure 5: Simulated 33] (black) and calculated [34] (red) magnetic field strengths (a) along and (b) perpendicular to the trap axis, for the Helmholtz-like configuration used in the EBIT [30. The relative magnetic field strengths are normalized to the maximum value, which occurs along the trap axis at the coils. The arrows in panel (b) indicate the radial location of the photon detectors used for decay spectroscopy.

with $0.25 \mathrm{~mm}$ thick, $>99 \%$ pure, pinhole-free Be windows to provide vacuum isolation $4^{4}$ A separate Be foil $(0.08 \mathrm{~mm})$ is located $22.5 \mathrm{~cm}$ radially from the trap center on the internal heat shield of the trap (Fig. 6(b)). The access ports corresponding to the large slits in the electrode-housing cylinder (Fig. 6(a)) are located at $90^{\circ}, 180^{\circ}$, and $270^{\circ}$ relative to the cryo-cooler at the top of the magnet housing, while the small slits are at $45^{\circ}, 135^{\circ}, 225^{\circ}$, and $315^{\circ}$. The port locations and the numbering convention used for them are displayed in Fig. 7 .

\section{Planar Si(Li) Detectors}

Each of the seven access ports around the EBIT houses a lithium-drifted silicon detector $(\mathrm{Si}(\mathrm{Li})$ ) (Fig. 8), which have good resolution and high efficiency at low photon energies $(<50 \mathrm{keV})$ [38]. These detectors were chosen over high-purity germanium (HPGe) crystals due to their decreased X-ray escape peak intensity $5^{5}$ and the prospect

\footnotetext{
${ }^{4}$ The EBIT has an ultra-high vacuum (UHV) environment that is between $10^{-11}$ and $10^{-13}$ Torr.

${ }^{5}$ Roughly four orders of magnitude at $20 \mathrm{keV}$ 38.
}

of performing a high-sensitivity X-ray measurement on ${ }^{76} \mathrm{Ge}$ [6].

\subsection{Design specifications}

The detectors were designed and constructed by Canberra, and each contains a $5 \mathrm{~mm}$ thick $\mathrm{Si}(\mathrm{Li})$ crystal with $\mathrm{a}<0.2 \mu \mathrm{m}$ dead-layer and $2000 \mathrm{~mm}^{2}$ active surface area. Each crystal is located $7 \mathrm{~mm}$ from the front face of the detector, which consists of a $0.6 \mathrm{~mm}$ layer of carbon that acts as a vacuum and thermal shield for the $\mathrm{Si}(\mathrm{Li})$ crystal inside the detector (Fig. 9(a)).

The detectors are structurally supported using a custombuilt aluminum frame that surrounds the central plane of the EBIT, which is mounted at the base of the magnet housing. The current mounting point of the frame has been a source of mechanical vibrations from the EBIT compressor (Section 5.2), and may be reconfigured in the near future to mitigate this problem. The crystals are kept at liquid-nitrogen $\left(\mathrm{LN}_{2}\right)$ temperatures for operation. The $\mathrm{LN}_{2}$ is provided to the crystals by an individual dewer directly attached to the cryostat that is controlled by the 


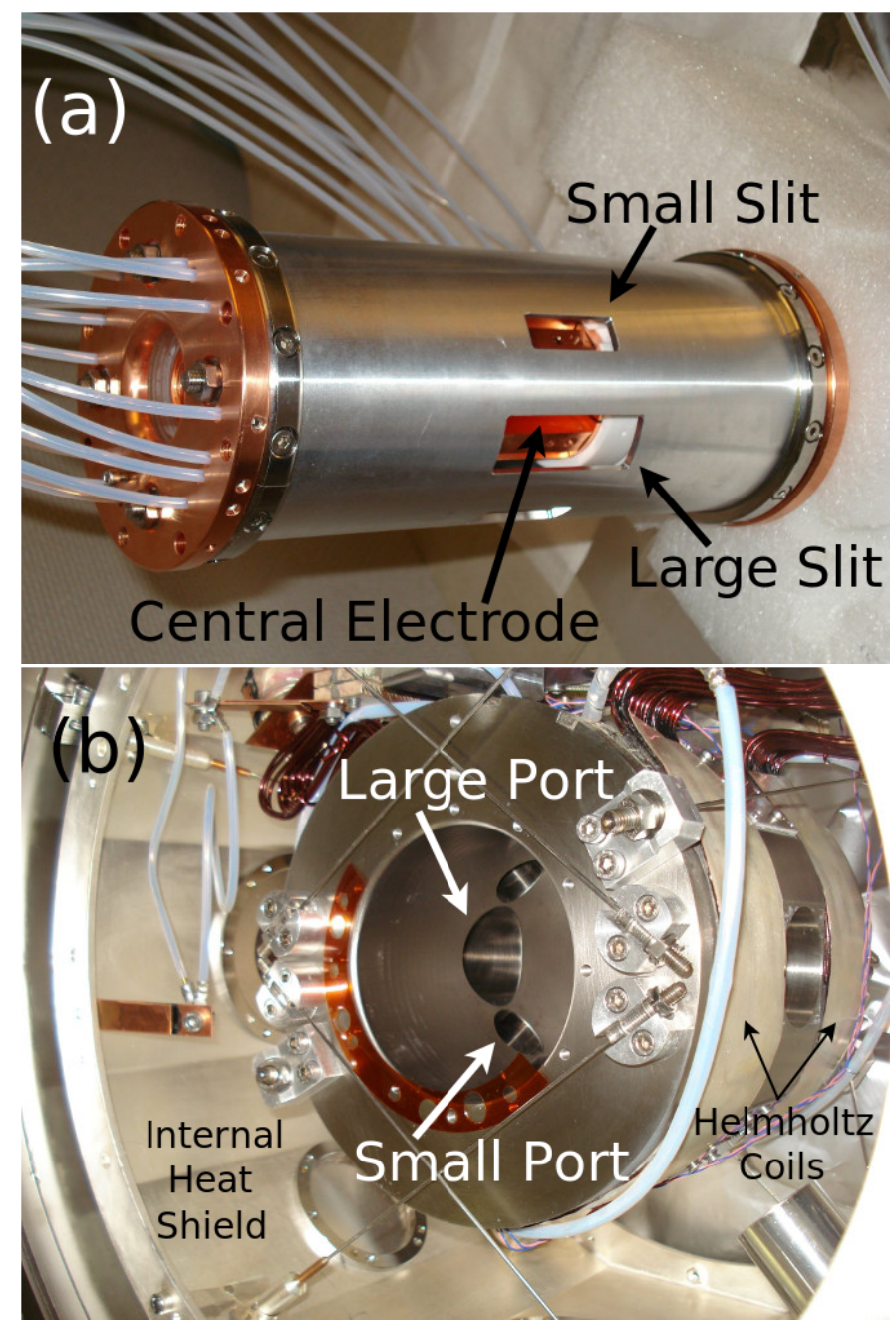

Figure 6: The primary components that comprise the interior of the TITAN EBIT. Displayed are: (a) both the eight-fold segmented central electrode (copper) and the housing cylinder (aluminum) that sit inside (b) the magnet coil holder. The solid-angle acceptance for photon detection from in-trap decays are limited by the slits in the electrode housing, as shown in panel (a).

\section{ISAC-EPICS [39] system.}

To reduce ambient background signals in the $\mathrm{Si}(\mathrm{Li})$ crystals, the outer casing of each detector is surrounded radially by $2 \mathrm{~mm}$ of ${ }^{n a t} \mathrm{Cu}$, followed by $1 \mathrm{~mm}$ of low-activity ${ }^{6}$ lead (Fig. 9). Lead $(Z=82)$ has a high photon interaction cross-section, which prevents radiation from entering the side of each detector, thereby reducing the overall ambient background contribution to the measured spectra by a

\footnotetext{
${ }^{6}$ The low-activity lead originates from the Britannia mine in Australia and features a ${ }^{210} \mathrm{~Pb}$ activity of $\leq 70 \mathrm{~Bq} / \mathrm{kg}$.
}

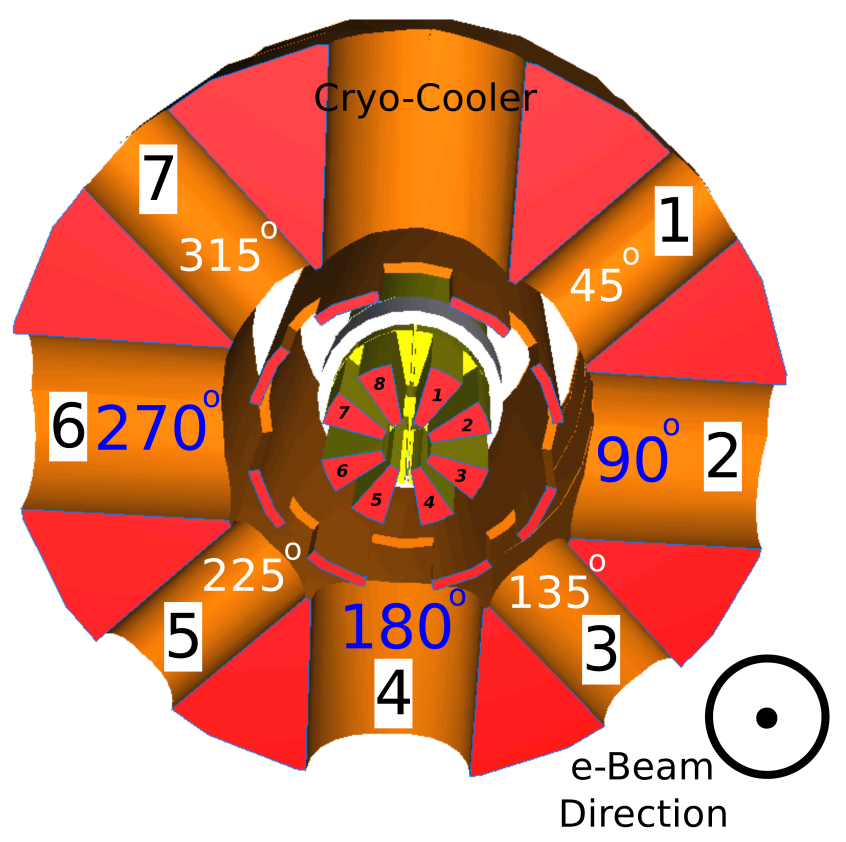

Figure 7: A cross-section of the GEANT4 [40] geometry for the magnet coil holder, eight-fold segmented central electrode, and housing cylinder, showing the locations for optical access to the trap center. The port angles are given in degrees for the large (blue) and small ports (white). All angles are shown relative to $0^{\circ}$, where the EBIT cryo-cooler is located. When facing the electron gun, the numbering convention for the seven open ports starts with \#1 at $45^{\circ}$ and moves clockwise around the trap.

factor of 3-4.

\subsection{Supplied power}

Each detector contains a Canberra PSC 854 transistorreset preamplifier, which provides a positive energy and timing output with a nominal impedance of $50 \Omega$. The PSC 854 preamplifier also contains an alarm card which provides notification in the event of a detector warm-up. The low-voltage power for the detector electronics and preamplifier is provided by a power supply which provides $\mathrm{DC} \pm 28 \mathrm{~V}$, while passively cooled linear voltage regulators provide voltages of $\mathrm{DC} \pm 12$ and $\pm 24 \mathrm{~V}$ separately for each detector. As a result, the stability of the voltage provided to the detectors is within $\sim 1 \mathrm{mV}$.

The crystals are biased to between -500 and $-600 \mathrm{~V}$ using an 8-channel ISEG EHS 8210x high-precision highvoltage (HV) power supply. The power-supply is con- 


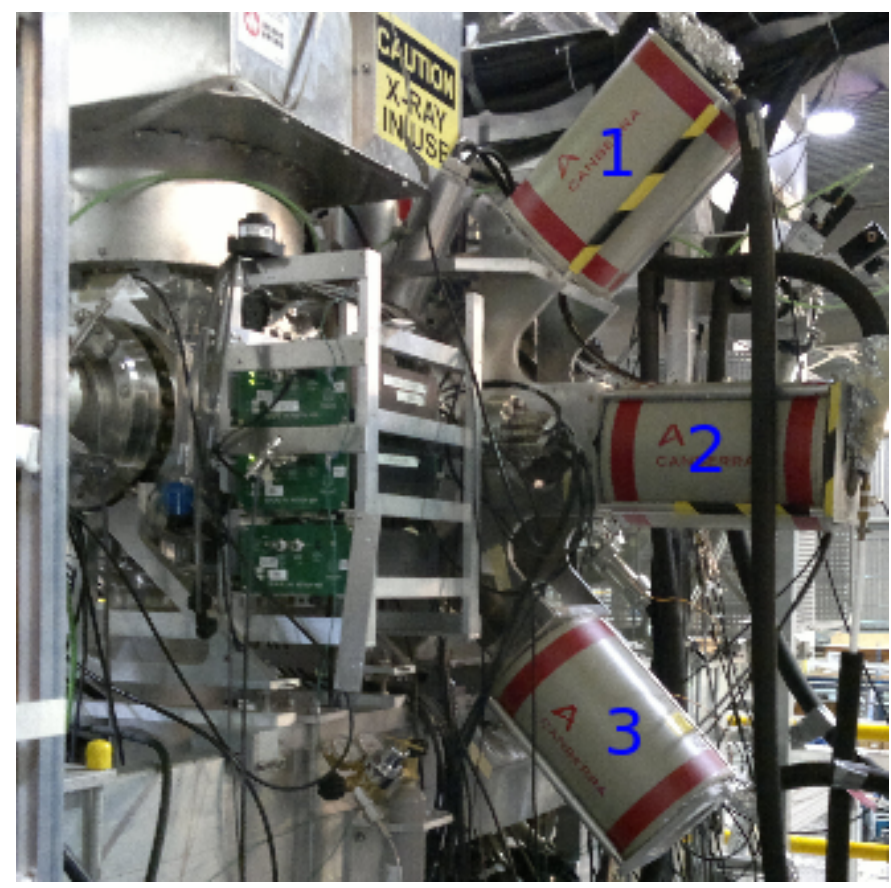

Figure 8: A photograph of $\operatorname{Si}(\mathrm{Li})$ detectors 1,2 , and 3 that surround the southern hemisphere of the EBIT. The $e$-collector is at the left of the image, where ion bunches from the RFQ are injected and extracted from the trap.

trolled via a CAN-interface, and has an auto-shutdown feature in case of a detector warm-up, which is connected to the HV control system. The detector preamplifier, HV, and data acquisition (DAQ) power supplies are all protected by a Tripp-Lite SU1000RTXLCD2U UPS backup system, which provides power conditioning with a $1 \mathrm{kVA}$ (900 W) on-line, double-conversion (pure sine wave) capability with an output voltage regulation of $\pm 2 \%$.

\subsection{Electronics and signal processing}

The preamplified signals from the detector are conditioned by a custom-built signal-processing amplifier before being digitized by an analogue-to-digital converter (ADC). The custom amplifier decouples the $4 \mathrm{~V}$ transistor-reset and filters the signal. The first stage is a high-pass filter, which allows the high-frequency, positive-step signal to pass through while removing the low-frequency ( $\sim$ few $\mathrm{Hz}$ ) noise from the compressor. Following this, the signal is amplified and integrated by a low-pass filter before

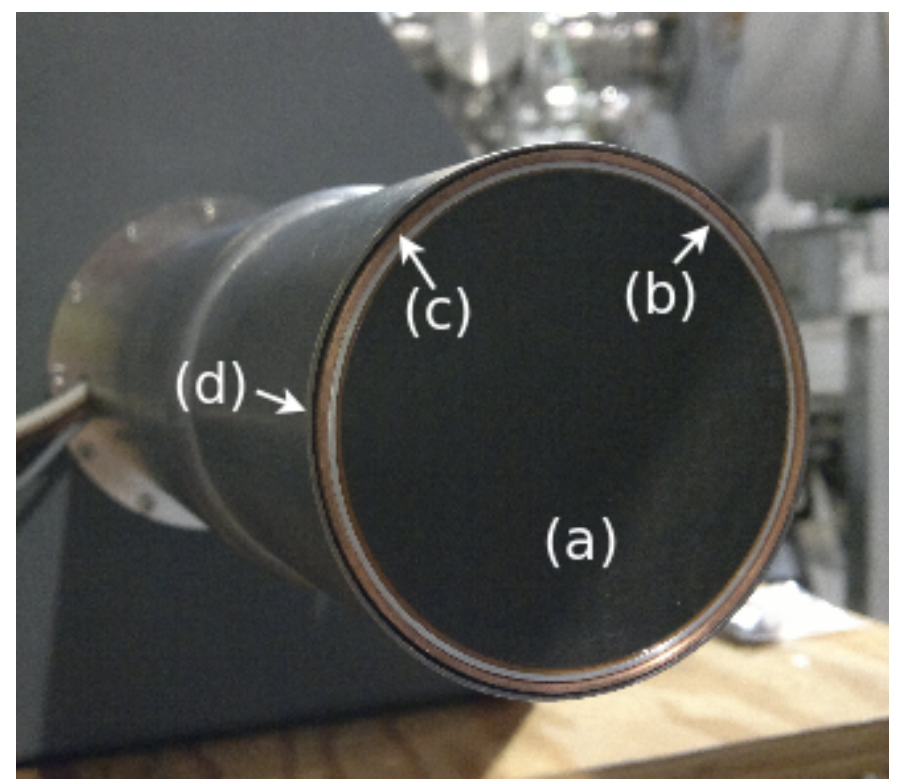

Figure 9: A view of the front face of a planar $\mathrm{Si}(\mathrm{Li})$ detector showing a) the thin carbon window, b) the detector's aluminum housing, c) the $\mathrm{Cu}$ absorber, and d) the $\mathrm{Pb}$ background shields.

digitization.

After processing, the signal digitization is performed by a self-triggered, 8-channel SIS3302 FPGA-based sampling ADC, clocked at $100 \mathrm{MHz}$ [41]. For each channel, the trigger threshold and required pulse shape (or rise time) are set individually. The ADC hardware applies a trapezoidal energy filter to generate a moving-average window with adaptable parameters that are used to approximate the pulse integration [41]. Once triggered, each event is recorded with a 48-bit time-stamp generated by the ADC clock, which is offset by a programmable pulse-generator (PPG) signal provided by the EBIT, thus providing a time for each event relative to the start of each measurement cycle.

Up to $65 \mathrm{k}$ samples of the pulse shapes are recorded per event, and can include pre-trigger samples for an off-line pulse shape analysis (PSA). This allows for the removal of invalid signals caused by noise and false triggers for the reduction of background events, as well as filtering and fitting valid pulses to improve the spectral resolution. 


\section{Environmental Effects}

The TITAN facility is located in a non-temperaturecontrolled environment, located roughly 5 metres above the ISAC experimental floor. As a result, environmental parameters affecting the sensitivity of the $\mathrm{Si}(\mathrm{Li})$ detectors are continuously monitored. To accomplish this, several diagnostic components are situated in various locations around the experimental setup, including thermocouples, vibration sensors, optical-light sensors, and voltage monitors.

\subsection{Thermal instabilities}

The day/night temperature variations in the experimental hall were observed to be between 5 and $10^{\circ} \mathrm{C}$, with a maximum summer temperature near the EBIT approaching $35^{\circ} \mathrm{C}$. These thermal instabilities manifest themselves in gain drifts of the preamplifier electronics, which were observed to be $<1 \%$ and can be accounted for. The temperature dependence of the ADC channel is displayed in Fig. 10. The detector resolution and efficiencies were shown to be constant over this temperature range and are thus not affected by the thermal cycles. The ADC and amplifier are also located in a non-temperature controlled environment, which can reach $40^{\circ} \mathrm{C}$ in the summer and may also contribute to the observed gain shift. This effect can be corrected for, and does therefore not generate anomalously poor resolutions.

\subsection{Vibration-induced noise}

The EBIT employs a two-stage Gifford-McMahon helium cryo-cooler that keeps the superconducting magnet at temperatures of $<6 \mathrm{~K}[43$ ] using a liquid-helium-free system [30]. The cooling is performed by a compressor unit that supplies high-pressure He gas to the cold-head and re-compresses the returned gas. In this process, the compression cylinder generates low-frequency $(1.2 \mathrm{~Hz})$ vibrational noise, which is subsequently transferred to the EBIT through the cold-head. As mentioned in Section 4.2, the

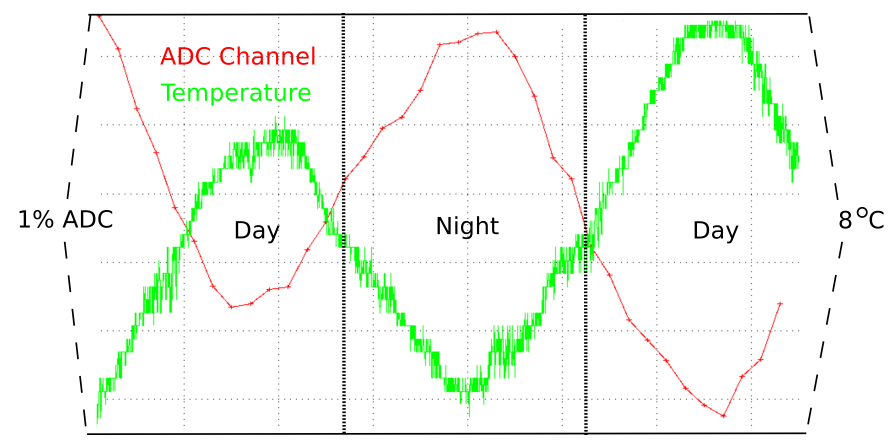

Figure 10: Observed thermal instability of the TITAN platform over the course of a 36 hour period (green), which shows a day/night temperature swing of nearly $8^{\circ} \mathrm{C}$. The drift in observed ADC channel number for the peak centroid of the $53 \mathrm{keV} \gamma$-ray from ${ }^{133} \mathrm{Ba}$ (red) shows a time-dependent fluctuation that is characteristic of the daily thermal cycle.

detectors are located in a frame that is directly mounted to the base of the magnet housing, which can transfer the vibrational noise generated by the He compressor directly to the $\mathrm{Si}(\mathrm{Li})$ detectors. The low-frequency noise does not pose a significant concern to the extracted signal due to the filtering process that is applied before digitization. However, these vibrations resonate at many frequencies in the aluminum detector-support frame and generate acoustic noise up to several-hundred $\mathrm{Hz}$. The distribution of highfrequency vibrations that exist at one of the horizontal access ports is displayed in Fig. 11, and shows significant noise at $\sim 120 \mathrm{~Hz}$ and $\sim 380 \mathrm{~Hz}$ which is only present when the compressor is on. The magnitude of this effect varies from detector to detector, and is correlated to the distance each port has from the frame mounting. A decrease in resolution by more than $20 \%$ at $50 \mathrm{keV}$ results from mounting the detectors directly to the Al frame, with no isolation. Several options for commercially available vibration-reduction and isolation materials are being explored to reduce this environmentally-induced noise.

\subsection{Magnetic field effects}

The effect of high magnetic fields on semiconductor photon detectors has been investigated previously [44, 45] 


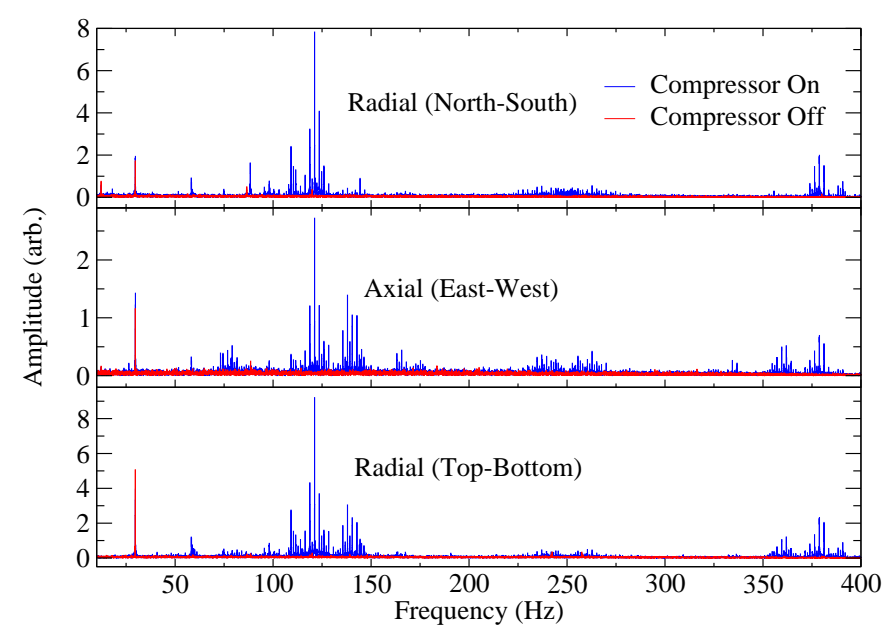

Figure 11: Observed vibrational frequencies from $10-400 \mathrm{~Hz}$ on the horizontal access ports at $90^{\circ}$ and $270^{\circ}$ when the cryo-cooler is on (blue) and off (red). The low-frequency region has been omitted to highlight the induced vibrational resonances in the $\mathrm{Al}$ frame. These measurements result from a fast-Fourier-transform (FFT) performed on data acquired by a TinkerForge [42] inertial-measurement unit (IMU), which has 9 degrees of freedom and consists of a 3-axis accelerometer, magnetometer, and gyroscope.

for different detector geometries and orientations with respect to a constant magnetic field. For the geometry and magnetic field of the TITAN EBIT, the influence of the field was investigated using a HPGe detector [7, 46], and was found to be small. The radial field strength at the crystal location 7 is roughly $5 \%$ of the value at the trap center, as displayed in Fig. 5. Therefore, for a magnet setting of $6 \mathrm{~T}$, the field experienced by the $\mathrm{Si}(\mathrm{Li})$ crystals is roughly $0.3 \mathrm{~T}$, which is well below the limit for possible field-related effects [44, 45]. As confirmation, the effect on the $\mathrm{Si}(\mathrm{Li})$ detection efficiency at $53 \mathrm{keV}$ was investigated by increasing the magnetic field at the trap center from 0 $2.5 \mathrm{~T}$, and no statistically significant effect was observed. The spectral resolution at $53 \mathrm{keV}$ was also investigated under the same conditions, and was found to be constant to within $1 \%$, with slight variations in centroid position. These centroid shifts do not pose a problem, as the magnetic field of the EBIT decays by only $1.2 \%$ per week.

\footnotetext{
${ }^{7} 23.2 \mathrm{~cm}$ away from the trap center
}

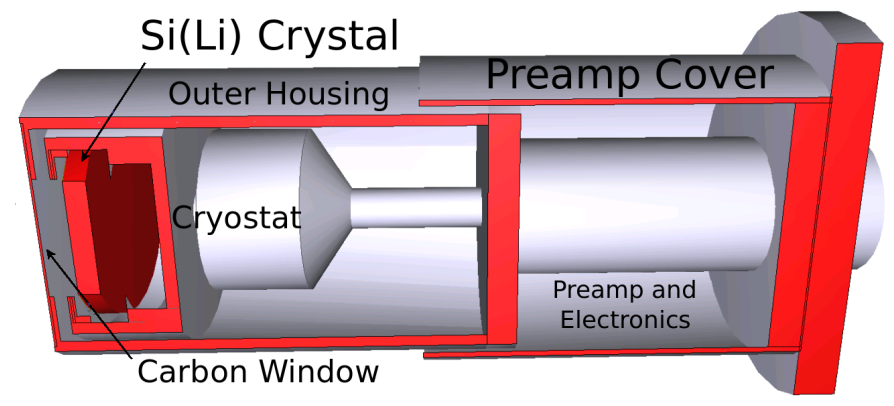

Figure 12: A cross-sectional view of the GEANT4 [40] geometry for one of the seven $\mathrm{Si}(\mathrm{Li})$ detectors. The geometry includes the $\mathrm{Si}(\mathrm{Li})$ crystal, structure housings, cold-finger, cryostat, and carbon window.

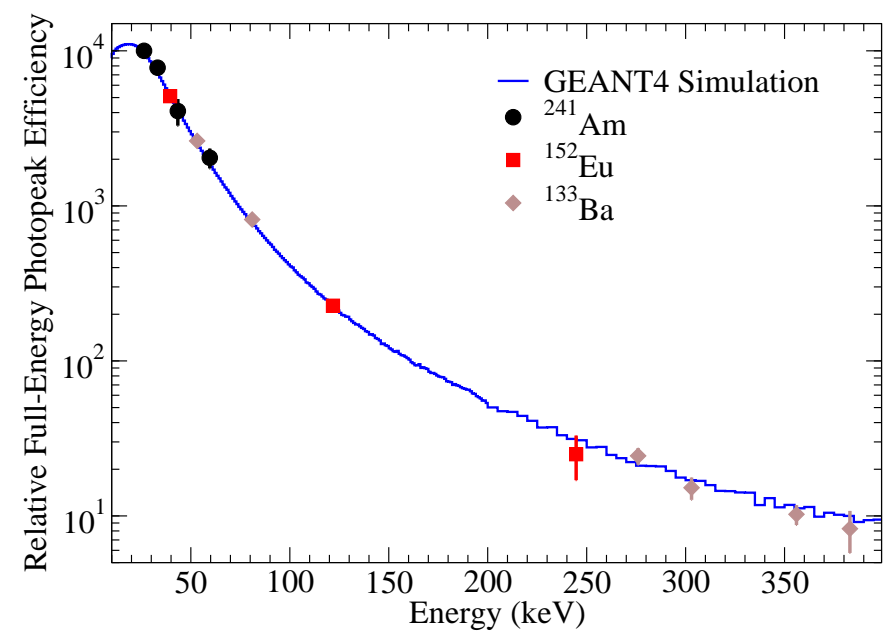

Figure 13: The experimental and simulated relative efficiency response as a function of energy for a typical $\mathrm{Si}(\mathrm{Li})$ detector. The experimental data were acquired using three different radioactive calibration sources when the $\mathrm{Si}(\mathrm{Li})$ detector was not mounted to the trap.

\section{GEANT4 Simulations}

One of the current limitations of this apparatus is a lack of access to the center of the trap for performing calibrations with radioactive sources. Therefore, detailed simulations are required to model the detector efficiencies during on-line running conditions. A GEANT4 [40] simulation was therefore developed to properly model the spectral response of the $\mathrm{Si}(\mathrm{Li})$ array surrounding the EBIT. The included geometries for both the trap and detector are displayed in Figs. 7 and 12 respectively. 
In addition to simulating radioactive source calibration data, a simulation of the realistic ion-cloud distribution is particularly important since the solid-angle acceptance differs due to the slit sizes in the electrode-housing cylinder (Fig. 6(a)). This variation in the access-port geometries generates a decrease in acceptance for the small ports of nearly a factor of 2 , leading to a $1.90(4) \%$ geometric acceptance for the full array.

Similar to the geometric acceptance, the intrinsic response of the crystals must be accurately reproduced by the simulation in order to generate the correct absolute efficiency of the array. This was accomplished by varying the carbon-window thickness and $\mathrm{Si}(\mathrm{Li})$ dead-layer in the GEANT4 geometry to match the observed crystal response from source measurements. A comparison of the experimental and simulated relative photopeak detection efficiencies for one of the $\mathrm{Si}(\mathrm{Li})$ detectors is displayed in Fig. 13. In order to extend this work for on-trap characterizations, the accurate reproduction of the two Be windows that separate the detectors from the trap center is also required.

Finally, to help with direct comparisons to RIB experimental data, the photon-energy deposition in the crystal is combined with the realistic $\mathrm{Si}(\mathrm{Li})$ response function that is derived from calibration measurements. These response functions include crystal imperfections that lead to slightly asymmetric photo-peaks due to incomplete chargecollection and trapped-charge effects 38]. Further on-trap benchmarking of these simulations was performed during the commissioning experiment and are presented in Section 7.

\section{Commissioning}

The commissioning of the decay-spectroscopy apparatus was performed using six $\mathrm{Si}(\mathrm{Li})$ detectors and one highpurity germanium (HPGe) detector in the seven access ports of the EBIT 8 In addition to testing the array with on-line radioactive beam, the goal of these measurements was to characterize and examine the capabilities of the setup by observing the EC decay of ${ }^{124}$ Cs [36, 37]. This case was chosen as the initial measurement due to its relatively large EC branching-ratio $(\approx 10 \%)$ and short half-life (31 s), which are both well known and therefore suited to providing a benchmark test.

The $A=124$ radioactive beam, consisting of ${ }^{124 g, m} \mathrm{Cs}$ and ${ }^{124 g, m}$ In, was delivered to the TITAN-RFQ where it was accumulated, cooled, and bunched for $1 \mathrm{~s}$, and subsequently transported at $1.5 \mathrm{kV}$ to the EBIT. The ions were stored for up to $25 \mathrm{~s}$ during the measurement portion of the cycle, at which point they were ejected and 5 s of trap-empty background was measured for comparison. The summed data over the course of the experiment for both portions of the measurement cycle from 15$130 \mathrm{keV}$ are displayed in Fig. 14, highlighting the observed $\mathrm{X}$ - and $\gamma$-ray lines from both ${ }^{124} \mathrm{Cs}$ and ${ }^{124} \mathrm{In}$. The resolution degradation caused by the vibration-induced noise described in Section 5.2 for the detectors used in the analysis was roughly $10 \%$, and did not pose a problem in resolving $\mathrm{X}$ - or $\gamma$-rays.

\subsection{Storage losses}

Previous decay measurements performed in the spectroscopy ion trap operated it in Penning trap mode, and replaced the $e$-gun with a passively-implanted planar-Si(Li) (PIPS) detector [7]. Although this facilitated the detection of charged-particle decay products for monitoring purposes, ions were continuously lost after injection due to poor confinement and residual gas interactions. The ion loss for Cs was studied in detail in Ref. [46, and it was found that roughly $90 \%$ of the ions were lost within the first $100 \mathrm{~ms}$ after ion-bunch injection. Therefore to overcome this problem the trap was operated in EBIT mode

\footnotetext{
${ }^{8}$ The HPGe detector was installed in port $5,32.5 \mathrm{~cm}$ away from the trap center.
} 


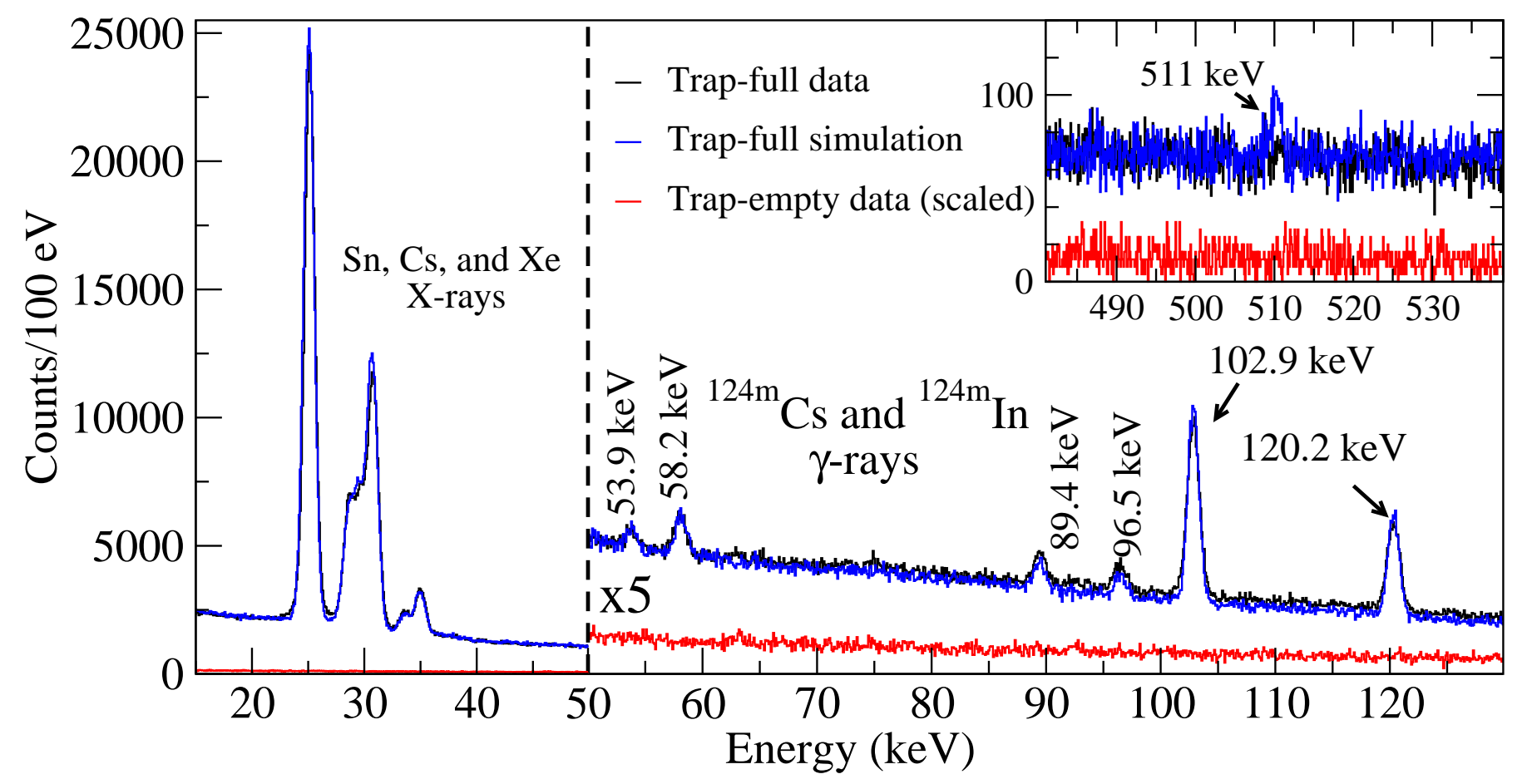

Figure 14: The observed photon spectrum from 15-130 keV, taken during the commissioning experiment, showing both the trap-full data (20 s/cycle) and simulation in black and blue, respectively. The trap-empty data $(5 \mathrm{~s} /$ cycle) (red) is representative of the ambient photon background, and has been scaled up by a factor of 4 for a direct comparison to the trap-full data. The complete removal of ions during the extraction phase of the cycle is demonstrated by the absence of X-and $\gamma$-ray lines in the trap-empty background spectrum. The inset displays the energy region from $480-540 \mathrm{keV}$, highlighting the absence of the $511 \mathrm{keV}$ positron annihilation radiation relative to a GEANT4 simulation with no $\vec{B}$ field.

by using a $50 \mathrm{~mA}$ e-beam, thereby exploiting the improved ion-injection, increased space-charge limit, and deeper potential well for longer and more stable trapping. The improved trapping is demonstrated in Ref. 37. for the typical cycle times of $\approx 30 \mathrm{~s}$; however, longer runs were also performed which showed trapping times of a minute or more with no observed ion losses.

The use of the $e$-beam also helped to improve the ionbunch injection and extraction, which is demonstrated in Fig. 14 by the observed trap-empty radiation. If poor injection caused ions to be spilled in the trap, or if the extraction was incomplete, the trap-empty portion of the cycle would show $\mathrm{X}$ - or $\gamma$-ray lines characteristic of ${ }^{124} \mathrm{Cs}$ decay.

\subsection{Positron-annihilation background suppression}

The decay-particle trajectories described in Section 3.2 also serve to increase the background reduction, as $511 \mathrm{keV}$ annihilation photons are significantly reduced due to the removal of charged decay particles from the trap center. For ${ }^{124} \mathrm{Cs} \beta^{+}$decay, there are nearly four times as many $511 \mathrm{keV}$ annihilation photons as $354 \mathrm{keV} \gamma$-rays [4]. As a result, a direct comparison of the efficiency corrected photopeak areas at $354 \mathrm{keV}$ and $511 \mathrm{keV}$ provide an estimate for the positron-annihilation background suppression,

$$
S_{511}=\left(\frac{I_{511}}{I_{354}}\right)_{\text {lit. }}\left(\frac{N_{354}}{N_{511}}\right)_{\text {exp. }}\left(\frac{\epsilon_{511}}{\epsilon_{354}}\right)_{\text {sim. }}
$$

where $I$ is the relative peak intensity from the literature [47, and $\epsilon$ is the simulated absolute photo-peak detection efficiency. The ratio $\frac{N_{354}}{N_{511}}$ is the fraction of observed counts in 


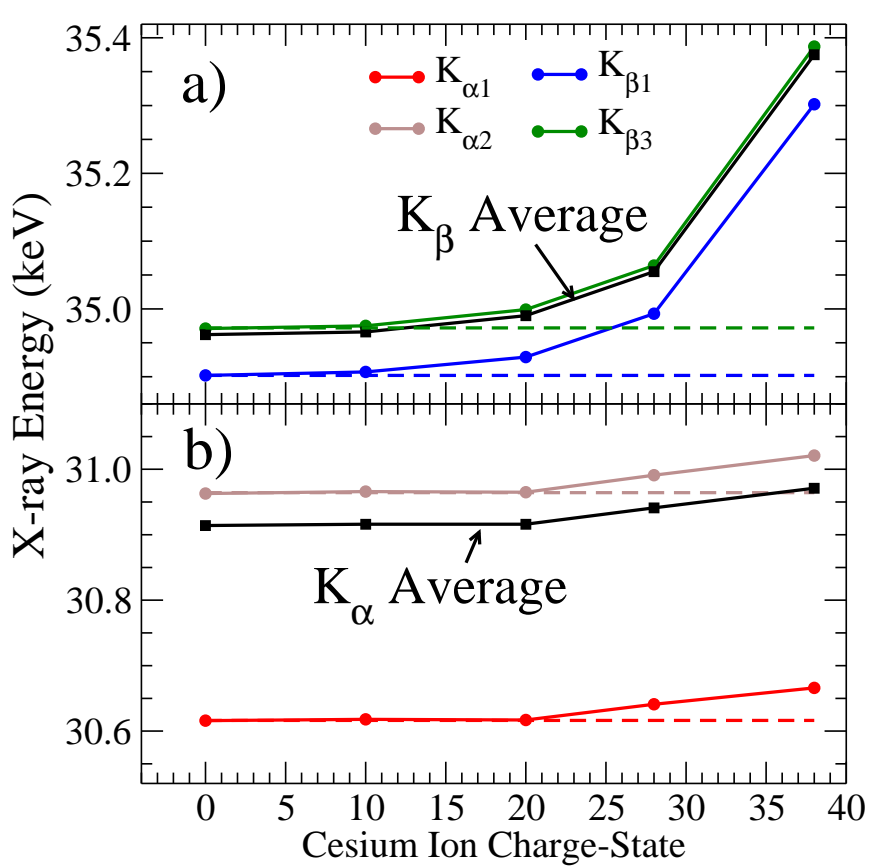

Figure 15: The theoretical change in a) $K_{\beta}$ and b) $K_{\alpha}$ X-ray energies as a function of charge-state for Cs ions. The calculations were performed using the multiconfigurational, relativistic DiracFock software, FAC [49], and display increasing $K_{\alpha}$ and $K_{\beta}$ energies as the charge-state increases. The dashed lines serve to illustrate the increasing energy difference relative to a neutral Cs atom. The weighted-average energy curves for $K_{\alpha}$ and $K_{\beta}$ result from the relative X-ray intensities, and represent what would be observed experimentally due to limited energy resolution.

the $354 \mathrm{keV}$ peak relative to the $511 \mathrm{keV} 1 \sigma$ upper limit. The observed limit on the background suppression was found to be a factor of 20 , which was also validated through a comparison of the experimental data to a GEANT4 simulation with no magnetic field (Fig. 14 (inset)).

\subsection{Atomic-structure effects}

Although charge breeding provides significant technical advantages for ion manipulation and storage [37, the use of HCIs for X-ray spectroscopy provides its own challenges, since the atomic structure is altered in the process. As electrons are removed from each successive atomic orbital, the structure of the remaining electrons deviates further from the neutral-atom case [48] (Fig. 15). This effect was observed in the commissioning experiment, and the observed relative average X-ray energy shift for ${ }^{124} \mathrm{Xe}$ of $\left\langle K_{\beta}-K_{\alpha}\right\rangle=90(40) \mathrm{eV}$ was in good agreement with the calculated value of $92 \mathrm{eV}$. Furthermore, no $K_{\beta 2}$ X-rays were observed since the charge states of the trapped Cs ions $\left(\approx 28^{+}\right)$corresponded to a fully stripped $M$-shell [37]. A distribution of ions in various charge-states simultaneously exist in the trap, which is well understood and has been investigated previously for TITAN's EBIT [30. Additional atomic effects were also observed [37], including changes in the $K_{\alpha} / K_{\beta}$ ratio, and in all cases were found to be consistent with theoretical calculations.

\subsection{Comparison to simulation}

Benchmarking of the GEANT4 simulations to in-trap data was also performed using the commissioning-experiment data. A comparison of the simulated and observed spectra is shown in Fig. 14 for the 15-130 keV photon energy region. Since the exact in-trap contributions of each species can vary from experiment to experiment, these quantities must be derived from the observed spectra. As a result, the simulated decay spectra are individually scaled and combined with an ambient-background spline function derived from the trap-empty measurements. The total MonteCarlo spectrum (blue) that results from this procedure exhibits general agreement with the data.

\section{Upgrades and Improvements}

\subsection{Multiple ion-bunch stacking}

For RIBs from ISAC with yields $\geq 10^{6} \mathrm{~s}^{-1}$, the limiting factor for ion-storage in the EBIT is the space-charge of the RFQ $\left(\sim 10^{5}-10^{6}\right)$ [52]. For nuclei with small branching ratios $\left(<10^{-4}\right)$, this limit would exclude the possibility of performing statistically significant measurements within a reasonable amount of time. As a result, a method for overcoming this space-charge limit was recently tested using a beam of ${ }^{116 g, m}$ In [53, 54], using short ( $\approx 10 \mathrm{~ms}$ ) RFQ accumulation times, with the subsequent injection of many ion 
bunches into the EBIT without extraction [55]. By using this multiple-injection technique, it was possible to stack several hundred ion-bunches in the EBIT, thus allowing for significantly more ions to be stored in the trap for one decay cycle. Having demonstrated this technique for the TITAN EBIT, several experiments that were previously unfeasible are now possible.

\subsection{Isobaric purification with the MR-ToF technique}

One of the advantages of manipulating ion-bunches in a multi-trap system is the possibility of isobaric cleaning. This form of beam purification has traditionally been performed with the assistance of a Penning trap, however this technique typically limits the total number of charges allowed to $\sim 10^{3}-10^{4}[56$ ]. The TITAN facility is currently in the process of adding a multi-reflection time-of-flight (MR-ToF) devic£ 9 wich can achieve ion capacities in excess of $10^{6}$ ions per bunch, while maintaining a mass resolving power of $\Delta m / m \geq 10^{5}$ [32]. This component will be included downstream from the RFQ, thus allowing for purification of SCIs before they are injected into the EBIT.

\subsection{Si $(\mathrm{Li})$ pulse-shape analysis (PSA)}

In an attempt to reduce the $\mathrm{Si}(\mathrm{Li})$ signal degradation caused by the vibrational effects described in Section 4.2 , the $\mathrm{Si}(\mathrm{Li})$ pulse-shape information recorded by the ADC can be used to further filter the data. At the moment, this filtering process simply ensures that the ADC is triggered by a pulse with the correct overall profile and average polarity. Once this filtering has been performed, a variable-size moving-average-window is applied, which has been shown to improve the effective $\mathrm{Si}(\mathrm{Li})$ detector resolution by $\approx 15 \%$. A more sophisticated PSA which performs a fit to the individual pulses is currently under development.

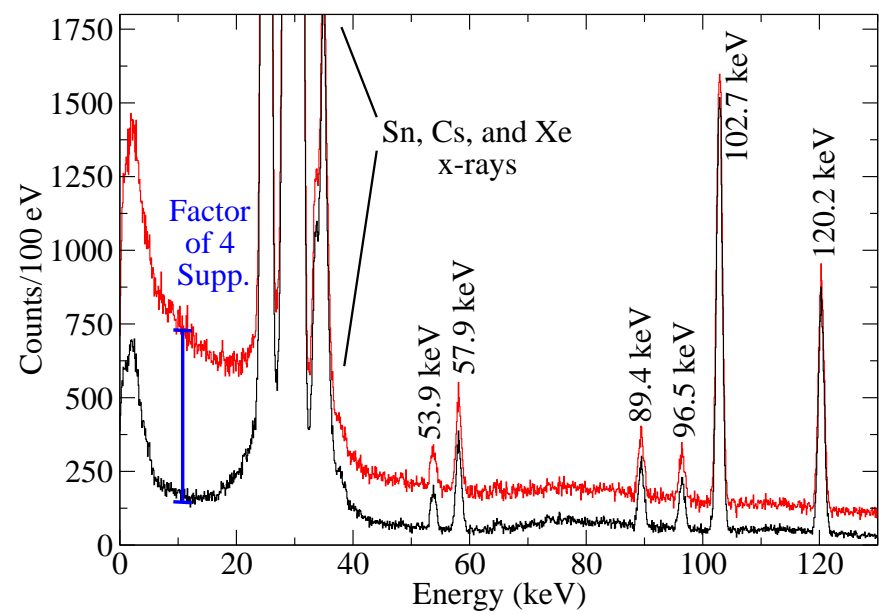

Figure 16: A GEANT4 40] simulation showing the effect of active Compton suppression with BGO shields, as described in the text. The simulated curves results from the same number of events from one detector during the commissioning ${ }^{124} \mathrm{Cs}$ experiment, where the red curve is the result for passive $\mathrm{Cu}$ and $\mathrm{Pb}$ shields only, and the black line is Compton suppressed photon spectrum using $1 \mathrm{~cm}$ of BGO surrounding the detector. The blue line demonstrates the factor-of-four Compton background suppression at $10 \mathrm{keV}$.

\subsection{Active Compton suppression}

The most common method for active Compton background suppression uses a scintillating material with a high $Z$ that surrounds the photon detector. This method improves the effective signal-to-background ratio by vetoing events with incomplete energy deposition in the $\mathrm{Si}(\mathrm{Li})$ crystal. The inorganic scintillating crystal Bismuth Germanium Oxide (BGO) is under investigation for active Compton suppression 38]. To collect the scintillation light from the BGO crystals, photomultiplier tubes (PMTs) are typically used. However, PMTs are unable to operate in the fringe magnetic fields around the TITAN EBIT (up to $\sim 0.15 \mathrm{~T})$. Therefore, development has begun to couple the BGO crystals to silicon photo-multipliers (SiPMs), which are able to operate in a high-field environment. The active shielding will be implemented in the future, and will provide a factor of 2-4 suppression of Compton-generated

\footnotetext{
${ }^{9}$ This device has been designed and constructed at Universität Gießen 32 and will be delivered to TRIUMF in the autumn of 2014 .
} 


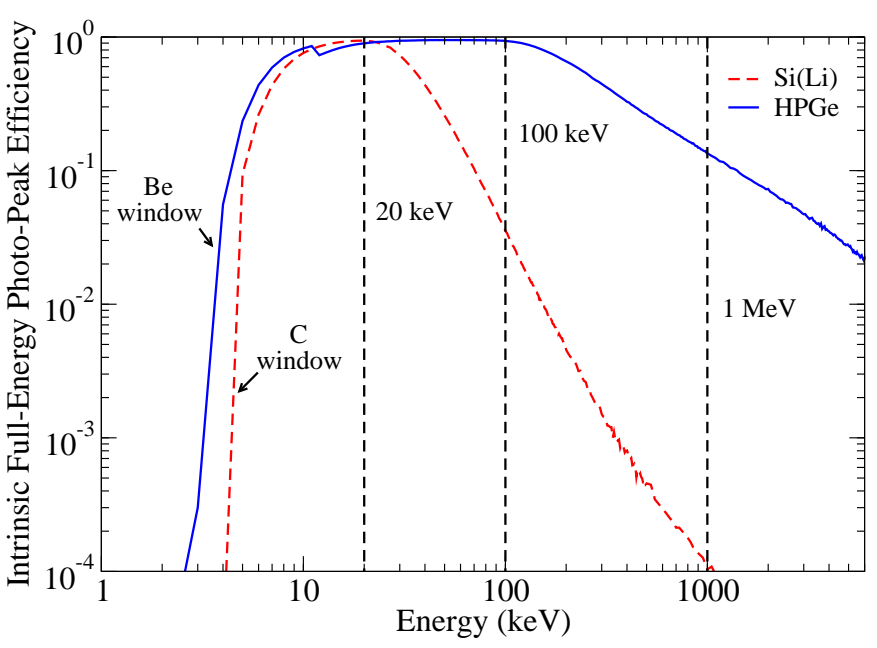

Figure 17: A comparison of the simulated intrinsic efficiencies for a TITAN $\mathrm{Si}(\mathrm{Li})$ detector and an $8 \pi$ HPGe detector separated from the source by the EBIT Be windows. The efficiency profiles are nearly identical below $\sim 20 \mathrm{keV}$, but a large increase in detection efficiency above this can be gained from using HPGe. This energydependent response therefore increases the versatility of performing decay spectroscopy in-trap with the TITAN EBIT. The low-energy character of the respective curves is determined by the thickness of the $\mathrm{Be}$ and $\mathrm{C}$ front-face windows on the HPGe and $\mathrm{Si}(\mathrm{Li})$ detectors, respectively.

background at low energies (Fig. 16).

\subsection{High-purity germanium detectors}

Although the current photon detectors have a high lowenergy photon detection efficiency, it drops dramatically at roughly $30 \mathrm{keV}$ which limits the range of experiments that are possible. The experimental capabilities can be increased by using high-purity germanium (HPGe) detectors, thereby increasing the versatility of performing intrap decay spectroscopy with TITAN. With the recent decommissioning of the $8 \pi \gamma$-ray spectrometer [57, 58] from TRIUMF, the prospect of deploying up to seven of these detectors in the ports around the EBIT exists due to their compatible size and availability. Each individual detector is composed of a cylindrical HPGe crystal with a radius of $2.65 \mathrm{~cm}$, and a length of $6.0 \mathrm{~cm}$. The crystals are located in an LN2-cooled cryostat, which is heat- and vacuum-shielded by a thin Be window on the front-face.
This situation has already been modelled in a GEANT4 simulation, and a comparison of the simulated absolute efficiencies for the HPGe and $\mathrm{Si}(\mathrm{Li})$ crystals are displayed in Fig. 17. These detectors can be used together with the $\mathrm{Si}(\mathrm{Li})$ detectors for in-trap experimentation, and will provide complimentary photon detection capabilities.

\section{Summary and Conclusions}

In summary, a novel in-trap decay spectroscopy setup has been developed using the TITAN facility at TRIUMF. The apparatus consists of 7 low-energy planar $\mathrm{Si}(\mathrm{Li})$ detectors which surround the TITAN EBIT; an open-access charge-breeding ion trap with a magnetic field of up to $6 \mathrm{~T}$. The current goal of this new facility is to provide a low-background environment for the observation of weak EC branching ratios of the intermediate nuclei for $\beta \beta$ decay. The ion-trap environment allows for the detection of low-energy photons by providing backing-free storage, while simultaneously guiding charged decay particles away from the trap center via the strong magnetic field. When combined with the intense electron beam of the EBIT, the strong magnetic field provides excellent ion confinement, which allows for long storage times. Impact ionization induced by the electron beam can increase the typical charge-states of the trapped ions to such a level that changes to the atomic structures were observed via X-ray energy shifts and $K_{\alpha} / K_{\beta}$ ratio changes. Although these atomic-structure alterations are a byproduct of the improved ion storage, these effects could be exploited in the future for studies on $\beta$ decay of HCIs. The background reduction provided by the apparatus presented in this work represents a significant step towards measuring weak branching ratios of $10^{-4}$ or less. This new facility is therefore poised to make a significant impact in the field of low-intensity spectroscopy with ARIEL and ISAC at TRIUMF. 


\section{Acknowledgements}

TRIUMF receives federal funding via a contribution agreement with the National Research Council of Canada (NRC). This work was partially supported by the Natural Sciences and Engineering Research Council of Canada (NSERC), and the Deutsche Forschungsgemeinschaft (DFG) under grant FR 601/3-1. TDM and ATG acknowledge support from the NSERC PGS-M and CGS-D programs, respectively. The authors also thank Dave Morris and Chris Pearson for their assistance in setting up the ISACEPICS control and DAQ, as well as Leonid Kurchaninov and Fuluny Jang for their electronics-related expertise. Additionally, we thank the Simon-Fraser University machine shop and electronics shop, for the efforts in constructing the $\mathrm{Si}(\mathrm{Li})$ detector frame and signal-processing amplifier, respectively.

\section{References}

[1] V. Cirigliano, S. Gardner, B.R. Holstein, Prog. Part. Nucl. Phys. 71, 93-118 (2013).

[2] F.T. Avignone, S.R. Elliott, J. Engel, Rev. Mod. Phys. 80, 481 (2008).

[3] J. Schechter and J.W.F. Valle, Phys. Rev. D 25, 2951 (1982).

[4] E. Takasugi, Phys. Lett. B 149, 372 (1984).

[5] A.S. Barabash, Phys. Rev. C 81, 035501 (2010).

[6] D. Frekers, J. Dilling, and I. Tanihata, Can. J. Phys. 85, 57-75 (2007).

[7] T. Brunner, A. Lapierre, C. Andreoiu, M. Brodeur, P. Delheji, S. Ettenauer, D. Frekers, A.T. Gallant, R. Gernhäuser, A. Grossheim, R. Krücken, A. Lennarz, D. Lunney, D. Mücher, R. Ringle, M.C. Simon, V.V. Simon, S.K.L. Sjue, K. Zuber, and J. Dilling, Eur. Phys. J. A 49, 142 (2013).

[8] Y. Blumenfeld, T. Nilsson, and P. Van Duppen, Phys. Scr. T152, 014023 (2013).

[9] R. Dunlop, G.C. Ball, J.R. Leslie, C.E. Svensson, I.S. Towner, C. Andreoiu, S. Chagnon-Lessard, A. Chester, D.S. Cross, P. Finlay, A.B. Garnsworthy, P.E. Garrett, J. Glister, G. Hackman, B. Hadinia, K.G. Leach, E.T. Rand, K. Starosta, E.R. Tardiff, S. Triambak, S.J. Williams, J. Wong, S.W. Yates, and E.F. Zganjar, Phys. Rev. C 88, 045501 (2013).

[10] K. Blaum, J. Dilling, and W. Nörtershäuser, Phys. Scr. T152, 014017 (2013).
[11] A. Gorelov et al., Phys. Rev. Lett. 94, 142501 (2005).

[12] P.A. Vetter et al., Phys. Rev. C 77, 035502 (2008).

[13] X. Fléchard et al., Phys. Rev. Lett. 101, 212504 (2008).

[14] V.Yu. Kozlov et al., Nucl. Instrum. Meth. Phys. Res. B 266, 4515 (2008).

[15] G. Li, R. Segel, N.D. Scielzo, P.F. Bertone, F. Buchinger, S. Caldwell, A. Chaudhuri, J.A. Clark, J.E. Crawford, C.M. Deibel, J. Fallis, S. Gulick, G. Gwinner, D. Lascar, A.F. Levand, M. Pedretti, G. Savard, K.S. Sharma, M.G. Sternberg, T. Sun, J. Van Schelt, R.M. Yee, and B.J. Zabransky, Phys. Rev. Lett. 110, 092502 (2013).

[16] C. Couratin, Ph. Velten, X. Fléchard, E. Liénard, G. Ban, A. Cassimi, P. Delahaye, D. Durand, D. Hennecart, F. Mauger, A. Méry, O. Naviliat-Cuncic, Z. Patyk, D. Rodríguez, K. Siegién-Iwaniuk, and J.-C. Thomas, Phys. Rev. Lett. 108, 243201 (2012).

[17] R.M. Yee, N.D. Scielzo, P.F. Bertone, F. Buchinger, S. Caldwell, J.A. Clark, C.M. Deibel, J. Fallis, J.P. Greene, S. Gulick, D. Lascar, A.F. Levand, G. Li, E.B. Norman, M. Pedretti, G. Savard, R.E. Segel, K.S. Sharma, M.G. Sternberg, J. Van Schelt, and B.J. Zabransky, Phys. Rev. Lett. 110, 092501 (2013).

[18] C. Weber, P. Möller, and P. Thirolf, Int. Journ. Mass Spec., 270-276 (2013).

[19] M.S. Mehlman, P.D. Shidling, R.S. Behling, B. Fenker, D. Melconian, and L. Clark, Nucl. Instrum. Methods Phys. Res. A 712, 9 (2013).

[20] P. Bricault et al., Nucl. Instrum. Meth. Phys. Res. B 126, 231 (1997).

[21] M. Dombsky, D. Bishop, P. Bricault, D. Dale, A. Hurst, K. Jayamanna, R. Keitel, M. Olivo, P. Schmor, and G. Stanford, Rev. Sci. Instr. 71, 978 (2000).

[22] U. Köster, Eur. Phys. J. 15, 255 (2002).

[23] J. Dilling, R. Krücken, and G. Ball, Hyper. Inter. 225, 1 (2014).

[24] J. Dilling, R. Krücken, and L. Merminga, Hyper. Inter. 225, 235 (2014).

[25] J. Dilling, P. Bricault, M. Smith, et al., Nucl. Instrum. Methods Phys. Res. B 204, 492 (2003).

[26] J. Dilling, R. Baartman, P. Bricault, et al., Int. Journ. Mass Spec. 251, 198 (2006).

[27] M. Smith, L. Blomeley, P. Delheij, et al., Hyper. Inter. 173, 304 (2006).

[28] T. Brunner, M. Smith, M. Brodeur, et al., Nucl. Instrum. Methods Phys. Res. A 676, 32 (2012).

[29] M. Brodeur, V. Ryjkov, T. Brunner, et al., Int. Journ. Mass Spec. 310, 20 (2012).

[30] A. Lapierre, M. Brodeur, T. Brunner, S. Ettenauer, A.T. Gallant, V. Simon, M. Good, M.W. Froese, J.R. Crespo López- 
Urrutia, P. Delheij, S. Epp, R. Ringle, S. Schwarz, J. Ullrich, and J. Dilling, Nucl. Instrum. Methods Phys. Res. A 624, 54 (2010).

[31] B.E. Schultz, U. Chowdhury, V.V. Simon, C. Andreoiu, A. Chaudhuri, A.T. Gallant, A.A. Kwiatkowski, T.D. Macdonald, M.C. Simon, J. Dilling, and G. Gwinner, Phys. Scr. T156, 014097 (2013).

[32] W.R. Plaß, T. Dickel, and C. Scheidenberger, Int. Journ. Mass Spec. 349, 134 (2013).

[33] COMSOL, Finite element simulation tool kit.

[34] H. Stöcker, Taschenbuch der Physik, Verlag Harri Detsch (2000).

[35] David A. Dahl, Int. Journ. Mass Spec. 200, 3 (2000).

[36] A. Lennarz, T. Brunner, C. Andreoiu, A. Chaudhuri, U. Chowdhury, P. Delheij, J. Dilling, S. Ettenauer, D. Frekers, A.T. Gallant, A. Grossheim, F. Jang, A.A. Kwiatkowski, T. Ma, E. Mané, M.R. Pearson, B.E. Schultz, M.C. Simon, V.V. Simon, Hyper. Inter. 225, 157 (2014).

[37] A. Lennarz, A. Grossheim, K.G. Leach, M. Alanssari, T. Brunner, A. Chaudhuri, U. Chowdhury, J.R. Crespo López-Urrutia, A.T. Gallant, M. Holl, A.A. Kwiatkowski, J. Lassens, T.D. Macdonald, B.E. Schultz, S. Seeraji, M.C. Simon, C. Andreoiu, J. Dilling, and D. Frekers, Phys. Rev. Lett. submitted (2014).

[38] Glenn F. Knoll, Radiation Detection and Measurement. John Wiley and Sons Inc., $3^{r d}$ Edition (2000).

[39] R. Keitel, D. Bishop, D. Dale, H. Hui, S. Kadantsev, M. Leross, R. Nussbaumer, J. Richards, E. Stuber, and G. Waters, ICALEPCS99, 674 (1999).

[40] S. Agostinelli et al., Nucl. Inst. and Meth. Phys. Res. A 506, 250-303 (2003).

[41] SIS3302-14xx Firmware-Gamma User Manual, SIS GmbH, Version sis3302-M-1407-1-V131-gamma.doc as of 11.09.2009

[42] Tinkerforge GmbH, Stukenbrock, Germany. http://www.tinkerforge.com

[43] S.W. Epp, Ph.D. Thesis, Ruprecht-Karls-Universität Heidelberg, (2007).

[44] A. Sanchez Lorente et al., Nucl. Instrum. Methods Phys. Res. A 573, 410 (2007).

[45] M. Agnello et al., Nucl. Instrum. Methods Phys. Res. A 606, 560 (2009).

[46] T. Brunner, Ph.D. Thesis, Technische Universität München, (2011).

[47] J. Katakura and Z.D. Wu, Nucl. Data Sheets 109, 1655 (2008).

[48] Heinrich F. Beyer, H.-J. Kluge, Viacheslav Petrovich Shevelko, $X$-ray Radiation of Highly-Charged Ions

[49] M.F. Gu, Can. J. Phys. 86, 675 (2008).

[50] R.D. Deslattes, E.G. Kessler Jr., P. Indelicato, L. de Billy, E. Lindroth, J. Anton, J.S. Coursey, D.J. Schwab, C. Chang,
R. Sukumar, K. Olsen, and R.A. Dragoset (2005), Xray Transition Energies (version 1.2). [Online] Available: http://physics.nist.gov/XrayTrans [2014, February 14]. National Institute of Standards and Technology, Gaithersburg, MD.

[51] M.F. Gu, Private Communication.

[52] M.R. Pearson, Private Communication (2014).

[53] R. Klawitter et al., In Prep. (2014).

[54] K.G. Leach et al., ARIS2014 Conference Proceedings In Prep. (2014).

[55] M. Rosenbusch et al., Appl. Phys. B, DOI: 10.1007/s00340-0135702-0 (2013).

[56] G. Savard, St. Becker, G. Bollen, H.-J. Kluge, R.B. Moore, Th. Otto, L. Schweikhard, H. Stolzenberg, and U. Wiess, Phys. Lett. A 158, 247 (1991).

[57] C.E. Svensson et al., Nucl. Instrum. Methods Phys. Res. A 204, 660 (2003).

[58] G.C. Ball et al., Journ. Phys. G 31, 1491 (2005). 